\title{
Alcohol Use, Drinking Venue Utilization, and Child Physical Abuse: Results from a Pilot Study
}

\author{
Bridget Freisthler
}

Published online: 15 January 2011

(C) The Author(s) 2011. This article is published with open access at Springerlink.com

\begin{abstract}
A positive relationship between parents' drinking and child physical abuse has been established by previous research. This paper examines how a parent's use of drinking locations is related to physical abuse. A convenience sample of 103 parents answered questions on physical abuse with the Conflict Tactics Scale-Parent Child version (CTS-PC), current drinking behavior, and the frequency with which they drank at different venues, including bars and parties. Ordered probit models were used to assess relationships between parent demographics, drinking patterns, places of drinking, and CTS-PC scores. Frequent drinking, frequently going to bars, frequently going to parties in a parent's own home, and frequently going to parties in friends' homes were positively related to child physical abuse. The number of drinking locations was positively related to child physical abuse such that parents who report attending and drinking at more of these venues were more likely to be perpetrators of physical abuse. This suggests that time spent in these venues provides opportunities to mix with individuals that may share the same attitudes and norms towards acting violently.
\end{abstract}

Keywords Alcohol use $\cdot$ Physical abuse $\cdot$ Child maltreatment $\cdot$ Venue utilization

In $2006,1.9$ per 1,000 children had substantiated reports of child physical abuse (U.S. Department of Health and Human Services, Administration on Children, Youth and Families 2008). Substantiation occurs when a child welfare

\section{B. Freisthler $(\bowtie)$}

UCLA Department of Social Welfare,

3250 Public Policy Building, Box 951656, Los Angeles, CA 90095-1656, USA

e-mail: freisthler@publicaffairs.ucla.edu investigator concludes that there is enough evidence to say the abuse or neglect actually occurred. Thus, substantiated reports are likely to undercount the true incidence of child physical abuse. In fact, a general population survey by Straus et al. (1998) found that 49 per 1,000 parents reported perpetrating severe physical assault (e.g., slapping on the face, head or ears) and 614 per 1,000 parents reported engaging in minor assaultive behaviors toward their children (e.g., shook the child).

One contributing factor to child physical abuse is a parent's use of alcohol. Rates of child maltreatment, particularly physical abuse, are higher among individuals reporting heavy drinking (Berger 2005; Famularo et al. 1986; Murphy et al. 1991, Kelleher 1994; Sun et al. 2001). Kelleher et al. (1994) found that parents who were identified as alcohol dependent or alcohol abusers were 4.7 times more likely to physically abuse their children than matched controls. Researchers have found a positive relationship between child maltreatment and alcohol abuse (Famularo et al. 1986; Murphy et al. 1991; Sun et al. 2001), and alcohol-abusing parents are more likely to be reported multiple times to the child welfare system for child maltreatment (Wolock and Magura 1996). Parents with a diagnosed substance use disorder are more likely to be physically abusive, commit child neglect, and have a higher child abuse potential (Ammerman et al. 1999; Chaffin et al. 1996).

Although numerous studies demonstrate a positive relationship between heavy alcohol use and child physical abuse there has been no study considering whether use of various drinking places may put children at greater risk. People who choose different locations to drink or spend time may be subject to social influences that increase risks for problem behaviors. For example, previous research indicates that adults who report drinking more frequently at restaurants are more likely to drive while intoxicated 
(Gruenewald et al. 2002). Within this population drinking at restaurants increases the risk of drunken driving. Among college students, those who report drinking at bars are more likely to drink to intoxication (Demers et al. 2002; Harford et al. 2002). Thus drinking in bars for this population is related to overall riskier drinking patterns. It may also be the case that parents who frequent places where drinking is more likely (regardless of their own drinking behaviors) may also place children at risk for certain types of child maltreatment.

Treno et al. (2007) explicated two theories that may explain why choice of drinking venue utilizatin is related to assaults among adults, namely social influence and social selection, that may also help explain this relationship for assaults by parents toward their children. The social influence model suggests that alcohol outlets, particularly bars, signal that norms against violence are relaxed in those areas because greater densities of these outlets are related to other markers of social disorganization such as poverty and residential instability (Sampson and Raudenbush 2004). The social selection model states that individuals who have greater inclinations to perpetrate violence are more likely to choose drinking venues that allow them to mix with similarly behaviorally-oriented individuals (Gruenewald et al. 2006; Parker 1993). Here violent individuals search for those environments and individuals that support those behaviors, or at the least, sanction them. These authors found that increased alcohol-related aggression was positively related to drinking at bars, parties in other people's homes, and drinking at friend's homes (Treno et al. 2007). Although this study could not definitely identify which of these two theories contributed to their findings it does provide a framework with which to situate this current work. Given this framework and the Treno et al.'s (2007) findings, frequency of drinking at bars, parties at other people's homes, and at friend's homes would be positively related to committing child physical abuse.

Although parent's use of different drinking locations and its relationship to child physical abuse has not been studied directly, Freisthler et al. (2004) found that Census tracts with more bars per mile had higher rates of substantiated child neglect while those with more off-premise outlets per mile were related to higher rates of substantiated child physical abuse. They concluded that the relationship between the density of bars and off-premise outlets and specific types of child maltreatment may be related to drinking behaviors of parents. For example, parents who drink in bars may leave their children unattended thereby increasing supervisory neglect. Parents drinking at home (and obtaining alcohol via off-premise alcohol outlets) may be at risk for child physical abuse (Freisthler et al. 2004). These findings have been validated in ecological studies of referrals to investigation by Child Protective Services, substantiations of child maltreatment, entries into foster care, and child abuse injuries resulting in at least one overnight stay in the hospital when measured at the Census block group, Census tract, and zip code levels (Freisthler 2004; Freisthler et al. 2004, 2005, 2007). However, these used official reports of child maltreatment, were ecological in nature (i.e., used geographic areas as the unit of analysis), and with the exception of Freisthler et al. (2004), combined all types of child maltreatment. Thus, in order to distinguish outlet effects at the individual or couple level, there is a substantial need to pursue studies to specific patterns of outlet use at the individual level.

In terms of other attributes, venue use (e.g., bars, restaurants) for drinking activities varies by both sociodemographic characteristics and drinking behaviors. Married people tend to use bars less often than single people, and married people with children tend to use bars less often than that (Gruenewald et al. 1995). Married individuals drink at their own home more often than single individuals (Treno et al. 2000). On the other hand, heavier drinking married people use bars more often than single people or married couples without children (Treno et al. 2000). Not surprisingly, drinkers who use alcohol more frequently also drink in more locations (Treno et al. 2000).

It is suggested in this paper that some drinking locations, such as bars or drinking at parties, may place children at greater risk for physical abuse when parents choose to go to these venues or events more often. The research presented here advances the current literature by using a sample of parents to understand how frequent drinking and utilizing various drinking locations may increase risks for child physical abuse. Although there is no measure of drinking at these various locations, this study can provide insights into the ways in which drinking contexts and venue use may be modified to reduce child physical abuse.

\section{Method}

Subjects and Data Collection

A convenience sample of 103 parents were surveyed during October-November, 2006. Parents were recruited from a variety of local agencies, including a day care center, a health clinic, and a social service agency. An incentive of \$25 was given to parents who agreed to participate in the survey. The survey was kept anonymous and confidential and verbal consent was obtained. Due to the anonymity of the survey, the research team did not have to report parents to Child Protective Services who may admit to engaging in physically abusive acts toward their children. The survey was a self-administered pencil and paper survey that took 
approximately $30 \mathrm{~min}$ to complete and covered questions related to parenting behavior, alcohol use, context of drinking, and social support. For parents who had more than one child, they were asked to answer the questions that asked about parenting behaviors for the child who had the most recent birthday. The research protocol was approved by the Institutional Review Boards at both the Pacific Institute for Research and Evaluation and the University of California, Los Angeles.

\section{Sample Characteristics}

Table 1 provides information about the sample used for this study. Eighty-five percent of the respondents were female and $59 \%$ were married or living with a partner. On average, the respondents were 33 years of age and 2.13 adults and

Table 1 Sample demographics

\begin{tabular}{|c|c|c|}
\hline & $\% /$ mean $(\mathrm{SD})$ & $\mathrm{n}$ \\
\hline \multicolumn{3}{|l|}{ Parent's gender } \\
\hline Male & 14.6 & 15 \\
\hline Female & 85.4 & 88 \\
\hline Age & $32.33(8.69)$ & 95 \\
\hline \multicolumn{3}{|l|}{ Race/Ethnicity } \\
\hline African American & 34.0 & 35 \\
\hline Hispanic & 14.6 & 15 \\
\hline White & 19.4 & 20 \\
\hline Other race/Ethnicity & 18.4 & 19 \\
\hline Multiple races/Ethnicities & 13.6 & 14 \\
\hline \multicolumn{3}{|l|}{ Marriage Status } \\
\hline Married/Living with a partner & 59.2 & 61 \\
\hline Single & 33.0 & 34 \\
\hline Divorced/Widowed/Other & 7.8 & 8 \\
\hline \multicolumn{3}{|l|}{ Highest level of education } \\
\hline High School or less & 20.6 & 22 \\
\hline Some college, no degree & 33.6 & 36 \\
\hline Associate's or Bachelor's degree & 30.8 & 33 \\
\hline Post-graduate or above & 11.2 & 12 \\
\hline \multicolumn{3}{|l|}{ Living in home } \\
\hline Adults & $2.18(1.31)$ & 103 \\
\hline Children & $2.23(1.57)$ & 102 \\
\hline \multicolumn{3}{|l|}{ Household income } \\
\hline$\$ 15,000$ or less & 27.1 & 29 \\
\hline$\$ 15,001-\$ 40,000$ & 25.2 & 27 \\
\hline$\$ 40,001-\$ 80,000$ & 20.6 & 22 \\
\hline$\$ 80,001$ or more & 21.5 & 23 \\
\hline \multicolumn{3}{|l|}{ Past year alcohol use } \\
\hline None & 35.9 & 37 \\
\hline Frequent & 22.2 & 22 \\
\hline Infrequent & 40.4 & 40 \\
\hline
\end{tabular}

2.23 children were living in the home at the time of the survey. The majority of the respondents had at least some college.

\section{Measures}

The dependent variable is child physical abuse as measured by the Parent-Child Conflict Tactics Scale (Straus et al. 1998). Parents were asked nine questions about minor and severe physical assault on a child. The minor abuse included items on hitting a child on the bottom with something like a hairbrush or belt and pinching him or her. The severe physical assault questions included items on slapping the child on the face, head, or ears, and throwing or knocking the child down. Response categories refer to the number of times this has happened during the past year and range from "Never" to "more than 10 times". Internal consistency for these scales range from .55 to .70 and have shown both construct and discriminant validity in a general population telephone survey (Straus et al. 1998). The dependent variable was coded as 0 for those individuals who did not engage in any of the physically assaultive behaviors, 1 for those parents who reported they participated in only minor physically assaultive behaviors or 2 for those parents who reported engaging in severe physical assault. Individuals who reported engaging in both minor and severe assault were coded as a 2 .

Parents were asked about their current drinking behaviors. Specifically they were asked whether they drank once a day or more, nearly every day, once or twice a week, about once a month, less than once a month but at least once a year, the last drink was over 12 months ago, or never had a drink of alcohol. This variable was then recoded to those drinkers that drank at least once a month or "frequent" drinkers, those that drank at least once a year but less than once a month or "infrequent" drinkers, and abstainers.

Venue utilization was measured in terms of the frequency during the prior 28 days when respondents reported attending these venues where drinking occurred and selfreported drinking in these venues, including (1) at home (only for drinking, not attending), (2) in bars, (3) in restaurants, (4) at parties in friends' homes, and (5) at parties in their own home for frequent drinkers. Infrequent drinkers were asked how often they had consumed alcohol at these same venues during the preceding 365-day period. Both drinkers and non-drinkers were asked the number of times they attended each venue when alcohol was available and they were not drinking. These items have been developed and used in telephone surveys (e.g., Treno et al. 2000; Gruenewald et al. 2002) and have estimated testretest reliabilities from a recent survey of adult drinkers in California that are generally good $(0.62<r<0.74)$. These 
variables were then recoded to reflect whether or not participants never attended each location/venue, attended/ drank in each location at least monthly, or attended/drank less than monthly, but at least once during the past year indicating non-use, frequent use, and infrequent use of the venue. In addition the number of drinking locations attended by each person was calculated by summing the number of venues reported (0-4), and the number of drinking locations where each person drank (0-5). Only $13 \%$ of parents reported not utilizing any of the drinking locations while about $11 \%$ indicated that they had used all four locations. About $20 \%$ percent of parents only drank in one venue while about $5 \%$ drank in all five venues. Drinking at parties at friend's homes and drinking at your own home were most prevalent while parents were less likely to drink at bars and parties in their own homes (Table 2).

Table 2 Sample characteristics of venue utilization for both attending and drinking

\begin{tabular}{|c|c|c|c|c|}
\hline & \multicolumn{2}{|c|}{ Drank } & \multicolumn{2}{|c|}{ Attend } \\
\hline & $\%$ & $\mathrm{n}$ & $\%$ & $\mathrm{n}$ \\
\hline \multicolumn{5}{|c|}{ Number of drinking locations $(n=87)$} \\
\hline 0 & 36.8 & 32 & 12.9 & 11 \\
\hline 1 & 19.5 & 17 & 21.2 & 18 \\
\hline 2 & 17.2 & 15 & 25.9 & 22 \\
\hline 3 & 13.8 & 12 & 29.4 & 25 \\
\hline 4 & 8.0 & 7 & 10.6 & 9 \\
\hline 5 & 4.6 & 4 & & \\
\hline \multicolumn{5}{|c|}{ Own home $(n=92)$} \\
\hline Frequent & 21.7 & 20 & & \\
\hline Infrequent & 17.2 & 16 & & \\
\hline None & 60.9 & 56 & & \\
\hline \multicolumn{5}{|l|}{ Bars $(n=92)$} \\
\hline Frequent & 15.2 & 14 & 17.0 & 16 \\
\hline Infrequent & 10.9 & 10 & 14.9 & 14 \\
\hline None & 73.9 & 68 & 68.1 & 64 \\
\hline \multicolumn{5}{|c|}{ Restaurants $(n=93)$} \\
\hline Frequent & 16.1 & 15 & 46.8 & 44 \\
\hline Infrequent & 22.6 & 21 & 27.2 & 28 \\
\hline None & 61.3 & 57 & 21.4 & 22 \\
\hline \multicolumn{5}{|c|}{ Parties at friend's homes $(n=92)$} \\
\hline Frequent & 22.8 & 21 & 33.3 & 26 \\
\hline Infrequent & 18.5 & 17 & 38.7 & 36 \\
\hline None & 58.7 & 54 & 28.0 & 31 \\
\hline \multicolumn{5}{|c|}{ Parties at own home $(n=91)$} \\
\hline Frequent & 9.9 & 9 & 13.0 & 12 \\
\hline Infrequent & 9.9 & 9 & 26.1 & 24 \\
\hline None & 80.2 & 73 & 60.9 & 56 \\
\hline
\end{tabular}

Demographics

Six demographic variables were included as controls in each analysis: parent age, parent gender, parent race/ ethnicity, marital status, number of children in the home, and income. Parent age and number of children in the home were included as continuous variables. Parent gender was coded as $0=$ female and $1=$ male. Due to the small sample size, parent race/ethnicity was also recoded as a dichotomous variable where $1=$ white and $0=$ non-white. Categories for marital status included married, living with a partner, single, divorced, widowed or other. For this analysis married and living with a partner were codes as 1 (married) and single, divorced, widowed, and other as 0 (not married). Because of the small sample size, income was dichotomous variable indicating low income (where $1=$ income less than $\$ 15,000$ a year).

\section{Data Analysis}

Data were analyzed in a series of ordered probit analyses for past year alcohol use, frequency of attendance at drinking locations, and number of drinking venues utlized on past year child physical assault. Parent age, gender, race/ ethnicity, low income status, and number of children were included as control variables for each analysis. Ordered probit models (as opposed to multinomial probit models) are used when the dependent variable, although discrete, has a natural ordering (Greene 1993). In this case, the ordered probit model takes into account the fact that underlying the ordering is a continuous descriptor of the dependent variable and the random error associated with this is normally distributed. The model follows the following form:

$\mathrm{y}^{*}=\beta^{\prime} x+\varepsilon$

where $y^{*}$ is the latent continuous measure of child physical assault, $x$ is a vector of the independent variables in the model, $\beta$ is a vector of the coefficients, and $\varepsilon$ is the normally distributed random error term. Here $y^{*}$ is not observed but is coded as the following:

$$
\begin{aligned}
\mathrm{y} & \left.=0 \text { if } \mathrm{y}^{*} \leq 0 \text { (no physical assault }\right) \\
& \left.=1 \text { if } 0<\mathrm{y}^{*} \leq \mu_{1} \text { (minor physical assault }\right) \\
& \left.=2 \text { if } \mu_{1}<\mathrm{y}^{*} \leq \mu_{2} \text { (severe physical assault }\right)
\end{aligned}
$$

where the $\mu$ 's are unknown parameters to be estimated with $\beta$. In addition to estimating the probit model, marginal effects of the model are also estimated at the mean of the independent variable. Data were analyzed using LimDep 8.0 (Greene 2002). Due to the small sample size, significance is reported at $p<.10$ level. 


\section{Results}

\section{Prevalence}

Overall, 42.4\% (39 of 92 respondents) of the sample reported engaging in at least minor physical assault toward their child. With regards to severe physical maltreatment, $12.8 \%$ (12 of 94 respondents) reported engaging in those behaviors. Rates of minor assault are lower than national population estimates ( 424 per 1,000 vs. 614 per 1,000), but rates of severe physical assault are higher in the current sample (128 per 1,000 vs. 49 per 1,000; see Straus et al. 1998 for general population estimates). Forty-nine percent of past year drinkers reported engaging in child physical assault compared to $28.6 \%$ of non-drinkers who reported engaging in physically assaultive behaviors.

\section{Drinking Behavior}

The results in Table 3 show that frequent drinkers (i.e., drink at least once a month) were more likely to engage in physically assaultive behaviors towards their child when compared to non-drinkers. Infrequent drinking (i.e., drinking at least once in the past year but less than once a month) was not related to physically assaultive behaviors.

\section{Venue Utilization}

Attending more drinking locations (regardless of drinking behavior) was positively related to the severity of child physical assault. Parents who attended bars frequently (at least once a month), go to parties at friend's homes frequently, and attended parties in their own home frequently were more likely to physically assault their child. There was no statistically significant relationship between drinking venue utilization and child physical maltreatment for parents who went to these venues infrequently (i.e., less than monthly). Findings for frequency of drinking at each venue were similar to attending the locations. Specifically, parents who frequently drank at bars, parties in their own home and parties in their friend's homes were more likely to commit child physical abuse. Drinking at one additional location was also assessed (own home). Parents reporting frequent drinking at home (at least monthly) were more likely to report child physical abuse (results not presented, but are available upon request).

\section{Marginal Effects}

The marginal effects (provided in Table 4) show similar results to those found in the overall model. More specifically, the table shows that frequent drinkers are about $25 \%$ more likely to commit minor physical abuse and about $18 \%$ more likely to commit severe physical abuse. Attendance at each additional drinking location significantly increases the probability of minor physical abuse by $6.2 \%$. Frequent attendance at bars increased the probability of minor physical abuse by about $12 \%$ and severe violence by $31 \%$. Significant marginal effects were also found for all levels of the dependent variable (no physical abuse, minor, and severe) for attending parties at your own home and parties at friends' homes.

\section{Discussion}

A considerable portion (about $40 \%$ ) of the study participants reported in engaging in at least minor physical violence toward their children. Being a current drinker, frequently attending bars, parties in friend's homes, and parties in other people's homes were all related to parents acting physically abuse towards their children. Similarly, drinking at multiple locations was positively related to child physical abuse. The findings were similar whether individuals drank at or just attended these locations. This suggests that even if parents choose not to drink at these locations, choosing to spend time in these venues provide them opportunities to mix with individuals who may share the same attitudes and norms towards violence. Treno et al. (2007) also found that adults who drink at bars and parties in friends' homes measured higher on alcohol-related aggression. Unlike Treno et al. (2007), however, this study examined the effects of attending these locations, regardless of drinking behaviors.

Previous research on venue utilization has shown that parents tend to use these venues less often then those without children (Gruenewald et al. 1995), but heavier drinking individuals (regardless of marital status) frequent bars more often (Treno et al. 2000). The current study suggests that even though, on the whole, parents may use these venues less often, frequent use of some of these venues by parents may place children at risk for physical abuse. That being said, what social mechanisms make these particular drinking contexts for parents potentially harmful to their children? Under most conditions parents will not participate in abusive behaviors but that may change under certain circumstances. One such circumstance may be that people who drink at bars or parties drink more and exhibit riskier drinking behaviors (Pihl et al. 1993, 1997; Treno et al. 2007). However, this study also found that going to these locations (bars, parties at friends' homes, and parties in your own home) regardless of drinking behaviors was related to committing physical abuse towards children. This further supports Treno et al.'s (2007) theory that some form of social influence or social selection effects may be at work. Here, parents who have aggressive tendencies may 
Table 3 Probit analysis of past year alcohol use, attendance at drinking locations, number of locations attended and child physical abuse
Each analysis is adjusted for age, gender, race/ethnicity, number of children, marital status, and income less than $\$ 15,000$

${ }^{+} p<.10, * p<.05, * * p<.01$, $* * * p<.001$

\begin{tabular}{lll}
\hline & Attend & \\
\cline { 2 - 3 } & $\mathrm{B}$ & $\mathrm{SE}$ \\
\hline Past year alcohol use $(n=82)$ & & $.3413^{* * *}$ \\
Frequent & 1.1825 & .4296 \\
Infrequent & -.0540 & $.1378^{*}$ \\
Drinking location & & \\
Number of Drinking Locations Attended $(n=71)$ & .3069 & $.3686^{* *}$ \\
Bars $(n=78)$ & & .3954 \\
Frequent & 1.1783 & .4114 \\
Infrequent & .2744 & .4230 \\
Restaurants $(n=77)$ & & \\
Frequent & .3318 & $.3494^{+}$ \\
Infrequent & .0367 & .3664 \\
Parties at friend's homes $(n=78)$ & & $.4191^{*}$ \\
Frequent & & .3689 \\
Infrequent & .5915 & \\
Parties at own home $(n=77)$ & -.4152 & .8798 \\
Frequent & & .0513 \\
Infrequent & & \\
\hline
\end{tabular}

choose venues or members for their social networks (e.g., friend's who also have parties) who condone violent actions or are influenced by other violent individual's who also frequent these locations.

These findings are somewhat contrary to findings on child maltreatment and alcohol outlet density. Freisthler et al. (2004) hypothesized that the positive relationship between off-premise density and child physical abuse may be due to the fact that these individuals are purchasing alcohol for use in their homes. They theorized that increased frequency of drinking in the homes may further lessen norms related to violence, resulting in abusive behaviors. That study, however, did not find a statistically significant effect of bar density on rates of physical abuse. Thus, the action of going to bars may have something to do with the overall disorganization of neighborhoods that exhibit less social control (both formal and informal) allowing violent behavior to occur (via the social influence model).

Although these findings are limited in their scope and generalizability there are some preliminary implications for changing environments. If bars are made safer (e.g., less aggressive), then perhaps the overall aggression at home and towards one's children after spending time in a bar would less likely result in lower levels of physical abuse. In fact, Gruenewald (2007) suggests that areas with greater densities of bars have a stratifying effect such that bar owners are better able to market to "niches" within the drinking crowd. This may have the effect of aggression or violent people drinking at the same location as to further reinforce those violent behaviors. Lowering densities of bars, according to this theory, may force "mixing" of populations, keeping violence and aggression more in check.

This would not, however, address the issue of increased physical abuse among parents who frequently attend parties at home or at friends' homes. Here the idea of "place managers" may be a viable option to reduce physical abuse. In the criminology literature, place managers have been described as individuals who discourage crime through their presence (e.g., security guards; Felson 1995). Place managers in the context of this study might include other adults whose job it is to care for and protect children (e.g., babysitters) especially when a parent is attending parties (Zimmerman 2007). It might even be that these parents need someone else to care for their child(ren) overnight, preventing them from being in immediate harm for risk. Greater neighborhood cohesion may facilitate this for parents (Sampson et al. 1999).

This study has not ruled out some alternate explanations for these findings. Parents who were abused and neglect as children (Berger 2005; Black et al. 2001), who report higher levels of depressive symptoms (Black et al. 2001), parenting stress, parent reactivity (e.g., impulsivity) and illicit drug use (Berger 2005) also tend to report higher levels of child maltreatment. This constellation of behaviors (e.g., impulsivity, parenting stress, mental illness) may serve as the impetus and/or major contributor to both frequent drinking and attendance or drinking at bars and parties. In other words, the relationship between some of the independent variables used here (e.g., frequent drinking, 
Table 4 Marginal effects of alcohol use and drinking venue utilization (comparing each group to the mean)

Type of Physical Assault

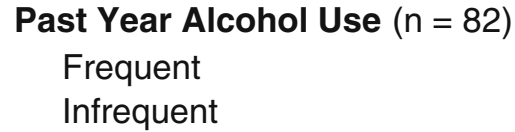

\section{Drinking Location}

Number of Locations Attended $(n=70)$

\author{
Bars $(n=76)$ \\ Frequent \\ Infrequent
Restaurants $(\mathrm{n}=76)$
Frequent
Infrequent
Parties at Friend's Homes $(n=76)$
Frequent
Infrequent
Parties at Own Home $(n=74)$
Frequent
Infrequent

\begin{tabular}{|c|c|c|c|c|c|}
\hline \multicolumn{2}{|c|}{ None } & \multicolumn{2}{|c|}{ Minor } & \multicolumn{2}{|c|}{ Severe } \\
\hline-.4396 & $* * *$ & .2513 & $* * *$ & .1883 & $* * *$ \\
\hline .0208 & & -.0136 & & -.0072 & \\
\hline-.1207 & * & .0621 & * & .0586 & \\
\hline-.4379 & $* \star *$ & .1254 & + & .3124 & $\star * *$ \\
\hline-.1083 & + & .0546 & & .0537 & \\
\hline $\begin{array}{l}-.1293 \\
-.0144\end{array}$ & * & $\begin{array}{l}.0656 \\
.0073\end{array}$ & & $\begin{array}{l}.0637 \\
.0071\end{array}$ & \\
\hline-.2317 & $* * *$ & .1124 & * & .1192 & + \\
\hline .1595 & * & -.0909 & *** & -.0685 & \\
\hline-.3374 & $* * *$ & .1152 & + & .2221 & * \\
\hline-.0202 & + & .0107 & & .0095 & \\
\hline
\end{tabular}

might shed further explicate the relationship between venue utilization and physical abuse but the small sample size in this study means the results could not be disaggregated for the analysis in this paper. As always, with a cross-sectional design, this study cannot make any inferences as to causality. Does drinking in bars or at friend's homes proceed physically abusive behaviors or is drinking a response to overall stress life events that results in abusive parenting? Finally, self-reports of physically abusive actions may be underreported or prone to social desirability bias. This survey did conduct preliminary analyses and found no correlation between a scale of social desirability bias and counts of physically abusive actions.

Despite these limitations, the numbers of parents selfreport physically abusive behaviors are higher that official reports and comparable to other general population estimates. This is also the one of the first studies to provide information on how venue utilization (i.e., bars or parties at friends' homes) might encourage or increase aggressive tendencies and therefore physically abusive behaviors of parents. Through understanding patterns of venue utilization for parents at risk for child physical, new prevention efforts that either address parent's drinking locations or parents commit minor and severe child physical assault 
provides some intervention at these locations may reduce child physical abuse. Similar to encouraging patrons to have designated drivers, encouraging parents to have suitable babysitters for their children may also become part of the norm at drinking establishments. Night time and overnight "drop-in" child care centers could be used to allow parents who drink heavily at venues have time to sober up before having to deal with child care responsibilities might reduce violence. Further, more specific measures of venue utilization could be constructed enabling a more precise understanding of this relationship. For example, are parents who go to bars and parties on the weekend or during late night hours more likely to engage in physically abusive parenting practices? Thus studies that continue to look at these and other environmental risk factors for both drinking and perpetration of child physical abuse might provide valuable information that aids in the prevention of child maltreatment if these environments can be modified.

Acknowledgements Research for and preparation of this manuscript were supported by a corporate development grant from the Pacific Institute for Research and Development, Calverton, MD and an NIAAA Center grant P60-AA06282.

Open Access This article is distributed under the terms of the Creative Commons Attribution Noncommercial License which permits any noncommercial use, distribution, and reproduction in any medium, provided the original author(s) and source are credited.

\section{References}

Ammerman, R. T., Kolko, D. J., Kirisci, L., Blackson, T. C., \& Dawes, M. A. (1999). Child abuse potential in parents with histories of substance use disorder. Child Abuse \& Neglect, 23, $1225-1238$.

Berger, L. M. (2005). Income, family characteristics, and physical violence toward children. Child Abuse and Neglect, 29, 107133.

Black, D. A., Heyman, R. E., \& Slep, A. M. S. (2001). Risk factors for child physical abuse. Aggression \& Violent Behavior, 6, 121188.

Chaffin, M., Kelleher, K., \& Hollenberg, J. (1996). Onset of physical abuse and neglect: psychiatric, substance abuse, and social risk factors from prospective community data. Child Abuse \& Neglect, 20, 191-203.

Demers, A., Kairouz, S., Adlaf, E. M., Gliksman, L., Newton-Taylor, B., \& Marchand, A. (2002). Multilevel analysis of situational drinking among Canadian undergraduates. Social Science and Medicine, 55, 415-424.

Famularo, R., Stone, K., Barnum, R., \& Wharton, R. (1986). Alcoholism \& severe child maltreatment. American Journal of Orthopsychiatry, 56, 481-485.

Felson, M. (1995). Those who discourage crime. In J. E. Eck \& D. Weisburd (Eds.), Crime and place: Crime prevention studies, vol. 4. Washington, DC: Police Executive Research Forum.
Freisthler, B. (2004). A spatial analysis of social disorganization, alcohol access, and rates of child maltreatment in neighborhoods. Children and Youth Services Review, 26(9), 307-319.

Freisthler, B., Midanik, L. T., \& Gruenewald, P. J. (2004). Alcohol outlets and child physical abuse and neglect: applying routine activities theory to the study of child maltreatment. Journal of Studies on Alcohol, 65(5), 586-592.

Freisthler, B., Needell, B., \& Gruenewald, P. J. (2005). Is alcohol and drug availability related to neighborhood rates of child maltreatment? Child Abuse and Neglect, 29(9), 1049-1060.

Freisthler, B., Gruenewald, P. J., Remer, L. G., Lery, B., \& Needell, B. (2007). Exploring the spatial dynamics of alcohol outlets and Child Protective Services referrals, substantiations, and foster care entries. Child Maltreatment, 12, 114-124.

Greene, W. H. (1993). Econometric Analysis. New York: Macmillan.

Greene, W. H. (2002). LimDep version 8.0. Plainview: Econometric Software Inc.

Gruenewald, P. J. (2007). The spatial ecology of alcohol problems: niche theory and assortative drinking. Addiction, 102, 870-878.

Gruenewald, P. J., Treno, A. J., Nephew, T. M., \& Ponicki, W. R. (1995). Routine activities and alcohol use: constraints on outlet utilization. Alcoholism: Clinical and Experimental Research, 19, 44-53.

Gruenewald, P. J., Treno, A. J., \& Johnson, F. (2002). Outlets, drinking and driving: a multilevel analysis of availability. Journal of Studies on Alcohol, 63, 460-468.

Gruenewald, P. J., Freisthler, B., Remer, L., LaScala, E. A., \& Treno, A. (2006). Ecological models of alcohol outlets and violent assaults: crime potentials and geospatial analysis. Addiction, 101, 666-677.

Harford, T. C., Wechsler, H., \& Seibring, M. (2002). Attendance and alcohol use at parties and bars in college: a national survey of current drinkers. Journal of Studies on Alcohol, 63, 726-733.

Kelleher, K., Chaffin, M., Holleberg, J., \& Fischer, E. (1994). Alcohol and drug disorders among physically abusive and neglectful parents in a community-based sample. American Journal of Public Health, 84, 1586-1590.

Murphy, J. M., Jellinek, M., Quinn, D., Smith, G., Poitrast, F. G., \& Goshko, M. (1991). Substance abuse and serious child mistreatment: prevalence, risk, and outcome in a court sample. Child Abuse and Neglect, 15, 197-211.

Parker, R. (1993). Alcohol and theories of homicide. In F. Adler \& W. S. Laufer (Eds.), New directions in criminological theory: Advances in criminological theory, vol. 4 (pp. 113-141). New Brunswick: Transaction.

Pihl, R. O., Peterson, J. B., \& Lau, M. A. (1993). A biological model of the alcohol-aggression relationship. Journal of Studies on Alcohol, 11, 128-139.

Pihl, R. O., Lau, M. L., \& Assaad, J.-M. (1997). Aggressive disposition, alcohol, and aggression. Aggressive Behavior, 23, 11-18.

Sampson, R. J., \& Raudenbush, S. W. (2004). Seeing disorder: neighborhood stigma and the social construction of 'Broken Windows'. Social Psychology Quarterly, 67, 319-42.

Sampson, R. J., Morenoff, J., \& Earls, F. (1999). Beyond social capital: spatial dynamics of collective efficacy for children. American Sociological Review, 64, 633-660.

Straus, M. A., Hamby, S. L., Finkelhor, D., Moore, D. W., \& Runyan, D. (1998). Identification of child maltreatment with the parentchild conflict tactics scales: development and psychometric data for a national sample of American parents. Child Abuse and Neglect, 22(4), 249-270.

Sun, A., Shillington, A. M., Hohman, M., \& Jones, L. (2001). Caregiver AOD use, case substantiation, and AOD treatment: 
studies based on two southwestern counties. Child Welfare, 80 , 151-177.

Treno, A. J., Alaniz, M. L., \& Gruenewald, P. J. (2000). The use of drinking paces by gender, age and ethnic groups: an analysis of routine drinking activities. Addiction, 95, 537-551.

Treno, A. J., Gruenewald, P. J., Remer, L. G., Johnson, F., \& LaScala, E. A. (2007). Examining multi-level relationships between bars, hostility, and aggression: social selection and social influence. Addiction, 103, 66-77.
U.S. Department of Health and Human Services, Administration on Children, Youth and Families. (2008). Child maltreatment 2006. Washington, DC: U.S. Government Printing Office.

Wolock, I., \& Magura, S. (1996). Parental substance abuse as a predictor of child maltreatment re-reports. Child Abuse \& Neglect, 20, 1183-1193.

Zimmerman, C. A. (2007). Routine activity theory and the handling of children and policymakers. Criminology and Public Policy, 6(2), 327-336. 